\title{
Blind Multiuser Deconvolution in Fading and Dispersive Channels ${ }^{1}$
}

\author{
Javier R. Fonollosał, José A. R. Fonollosa†, Zoran Zvonarłand Josep Vidal† \\ $\dagger$ Universitat Politècnica de Catalunya \\ Department of Signal Theory and Communications \\ \#Analog Devices \\ 08034 Barcelona, SPAIN \\ fono@tsc.upc.es \\ Communications Division \\ Wilmington MA 01887-1024, USA \\ Zoran.Zvonar@analog.com
}

\begin{abstract}
An adaptive near-far resistant technique for the blind joint multiuser identification and detection in asynchronous CDMA systems is analyzed in fading and dispersive GSM channels.
\end{abstract}

\section{INTRODUCTION}

Multiuser detection in CDMA systems usually requires either knowledge of the transmitted signature sequences and channel state information or use of a known training sequence for adaptation. Consequently blind adaptive multiuser receivers have gained considerable attention [1]. We recently proposed a joint multiuser deconvolution scheme [2] characterized by:

- No knowledge of timing, channel state information or signatures nor use of training sequences is required for any user.

- The estimate of the signature sequence of each user convolved with its physical channel impulse response is provided after initial convergence.

- The blind multiuser detector is near-far resistant.

The purpose of this paper is to further investigate the behavior of this scheme in fading and dispersive channels.

\section{SySTEM MODEL}

We consider the asynchronous CDMA channel

$$
r(t)=\sum_{n} \sum_{k=1}^{K} b_{k}[n] h_{k}(t-n T, t)+\sigma w(t)
$$

where $h_{k}(t-n T, t)$ is the overall complex channel impulse response, given by the convolution of the signature sequence, physical radio channel and the receiving filter impulse responses. It incorporates the amplitude and the delay for user $k$, and its duration is assumed to be smaller or equal to $L$ symbols, i.e. $h_{k}(\tau, t)=0, \tau<0, \tau>L T, \forall t$. The total number of active users is $K$ and their transmitted sequences are binary independent symbols $b_{k}[n] \in$ $\{1,-1\}$. The symbol rate is $1 / T$ and $w(t)$ is normalized white Gaussian noise. The CDMA channel is sampled at a rate $M / T=1 / T_{s}$ to derive the vector sequence $\mathbf{r}[n]$

$$
\mathbf{r}[n]=\left[r(n T), r\left(n T+T_{s}\right), \ldots, r\left(n T+(M-1) T_{s}\right)\right]^{T}
$$

The observation $\mathbf{r}[n]$ is modeled as a probabilistic $M$ length vector sequence of a state vector $\mathbf{s}[n]$

$$
\mathbf{r}[n]=\mathcal{H}[n] \mathbf{s}[n]+\mathbf{w}[n],
$$

\footnotetext{
${ }^{1}$ Work supported by CIRIT of Catalonia (GRQ93-3021).
}

where $(M \times K L)$ matrix $\mathcal{H}[n]$ depends of the overall discrete impulse response for all users and $\mathbf{w}[n]$ is the normalized noise vector. There are $N=2^{L K}$ possible state vectors corresponding to $L$ binary symbols of $K$ users.

\section{Blind Identification And Detection ALGORITHM}

If the overall impulse response for each user was known, that is if the signature sequence, physical channel impulse response, amplitude and delay corresponding to each user were available, then using this information, the Viterbi algorithm could be employed to determine the multiuser maximum-likelihood transmitted sequence. In the method we presented however, the Viterbi algorithm is applied with current estimates of the overall impulse responses which are updated recursively after arbitrary initialization. The number of users $(K)$ is assumed known together with a bound for the impulse response duration $(L)$. A similar approach was proposed for the blind equalization of single user channels using the Viterbi algorithm [3] and the Baum-Welch identification algorithm [4]. Specific to the multiuser approach is the procedure which overcomes the convergence to a local minimum [2].

\section{Behavior in Fading and Dispersive Channels}

The blind multiuser algorithm has been tested using the mobile radio channel model for typical urban areas (Type 1) TUX60, as defined in [5]. Simulations indicate that, for moderate Doppler frequency $(50 \mathrm{~Hz})$ and multipath spread ( 1.35 symbols), convergence can still be attained within few hundred symbols. Afterwards, the algorithm is still able to track slow channel variations. Possible modification of the receiver, after the initial convergence, may include a simpler decision-directed MMSE scheme.

\section{REFERENCES}

[1] S. Verdú, "Adaptive Multiuser Detection," Proc. Third International Symposium on Spread Spectrum Techniques and Applications, Oulu, Finland, pp. 43-50, July 1994.

[2] J. R. Fonollosa, J. A. R. Fonollosa, Z. Zvonar, and J. Vidal, "Blind Multiuser Identification and Detection in CDMA Systems," Proc. IEEE ICASSP-95, pp. 1876-1879, May 1995.

[3] N. Seshadri, "Joint Data and Channel Estimation using Blind Trellis Search Techniques," IEEE Trans. on Communications, vol 42, pp. 1000-1011, March 1994.

[4] J. A. R. Fonollosa and J. Vidal, "Application of Hidden Markov Models to Blind Channel Characterization and Data Detection," Proc. IEEE ICASSP-94, pp. IV 185-188, April 1994.

[5] GSM recommendation 05.05 (version 3.11.0). 